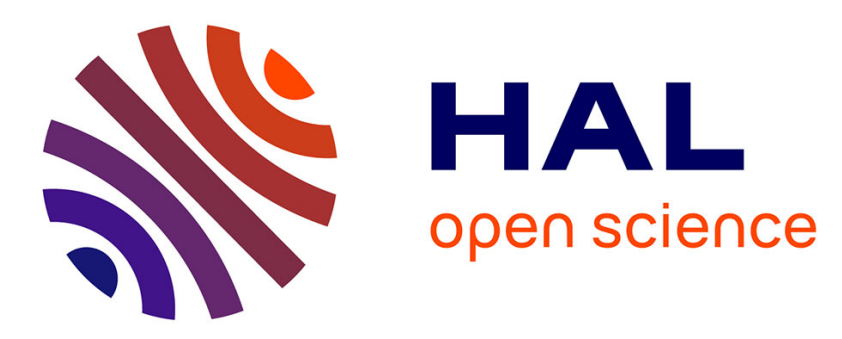

\title{
Un nouvel essai de classification des batu Aceh de la péninsule malaise
}

Daniel Perret, Kamarudin Ab. Razak

\section{To cite this version:}

Daniel Perret, Kamarudin Ab. Razak. Un nouvel essai de classification des batu Aceh de la péninsule malaise. Archipel, 2003, 66, pp.29-45. 10.3406/arch.2003.3782 . halshs-01883846

\section{HAL Id: halshs-01883846 \\ https://shs.hal.science/halshs-01883846}

Submitted on 28 Sep 2018

HAL is a multi-disciplinary open access archive for the deposit and dissemination of scientific research documents, whether they are published or not. The documents may come from teaching and research institutions in France or abroad, or from public or private research centers.
L'archive ouverte pluridisciplinaire HAL, est destinée au dépôt et à la diffusion de documents scientifiques de niveau recherche, publiés ou non, émanant des établissements d'enseignement et de recherche français ou étrangers, des laboratoires publics ou privés. 


\title{
Un nouvel essai de classification des batu Aceh de la péninsule
} malaise

\author{
Daniel Perret, Kamarudin Ab. Razak
}

\section{Abstract}

Daniel Perret

This article presents an attempt at classifying a family of old Moslem tombstones found in the Malay World, called batu Aceh because several elements point at North Sumatra as the origin of their artistic tradition. The corpus consists in 450 tombs found in the Malay Peninsula during surveys carried out since 1996. They are distributed in five groups according to the general shape of the main part of the tombstone. Each group includes one or more specific forms ( 20 on the whole). This classification is a tool of comparison intended to help the historical interpretation of the tombs in a context where inscriptions of a historical nature are very scarce.

\section{Citer ce document / Cite this document :}

Perret Daniel, Razak Kamarudin Ab. Un nouvel essai de classification des batu Aceh de la péninsule malaise. In: Archipel, volume 66, 2003. pp. 29-45;

doi : https://doi.org/10.3406/arch.2003.3782

https://www.persee.fr/doc/arch_0044-8613_2003_num_66_1_3782

Fichier pdf généré le 22/04/2018 


\section{Un nouvel essai de classification des batu Aceh de la péninsule malaise}

Dans le monde malais, les pratiques funéraires musulmanes s'enrichissent, dès la fin du XIII ${ }^{\mathrm{e}}$ siècle au moins, de stèles qui présentent des matériaux, des formes et des décors caractéristiques. Plusieurs éléments conduisent à penser que cette famille de stèles est le produit d'une tradition artistique qui prend naissance avec la diffusion de l'islam au nord de Sumatra, en particulier à Pasai et à Aceh, d'où l'expression «batu Aceh» donnée à ces monuments (1). Du point de vue chronologique d'abord, la majorité des stèles datées les plus anciennes proviennent de cette zone. Dans l'état actuel des connaissances, au premier rang de celles portant une date confirmée (Ramadhan 696 H. [1297 E.C.]) figure en effet celle du sultan Malik alSalih, le premier sultan du royaume de Sumatra-Pasai. Du point de vue quantitatif ensuite, de tout le monde malais, ce sont les régions de Pasai et d'Aceh qui en recèlent, de loin, le plus grand nombre. Même si nous manquons de données récentes et précises à ce sujet, les tombes à batu Aceh s'y comptent probablement par milliers. Vient ensuite la péninsule malaise où un peu plus de 400 tombes ont été recensées pour l'instant. L'aire de distribution s'étend en fait de la région de Pattani (2), au sud de la Thaïlande, jusqu'à

1. En péninsule malaise, l'expression «batu Aceh» semble apparaître en 1920 à propos d'une tombe de l'État de Pahang (Othman bin Mohd. Yatim, Batu Aceh. Early. Islamic Gravestones in Peninsular Malaysia, Kuala Lumpur, Muzium Negara, 1988, p. xxv, 5).

2. Seize tombes y sont encore visibles. Cf. D. Perret, «Tombes musulmanes anciennes et voies possibles d'islamisation de la région de Pattani », in Daniel Perret et al. (éds.), Études sur l'histoire du sultanat de Patani, Paris, EFEO, à paraître en 2004. 
la province de Sulawesi en Indonésie, couvrant notamment plusieurs provinces de Sumatra et de Java, ainsi que Brunei. Enfin, l'exportation de batu Aceh à partir d'Aceh vers la péninsule malaise est attestée dans un texte malais, le Bustan al-Salatin, rédigé en 1640 par Nuruddin al-Raniri (3).

Les nombreuses prospections effectuées depuis 1996 en péninsule malaise conjointement par l'École française d'Extrême-Orient et la Yayasan Warisan Johor (Fondation pour l'Héritage de Johor) afin d'y étudier spécifiquement les batu Aceh, permettent d'affirmer que, parmi les stèles funéraires musulmanes en pierre sculptée encore visibles, c'est de très loin la famille la mieux représentée dans cette région pour la période allant du milieu du XVe siècle à la fin du XVIII ${ }^{e}$ siècle. La variété de types ainsi que la richesse de décors sont également sans équivalent. Dans un contexte de rareté des vestiges musulmans anciens dans la région, les batu Aceh constituent par conséquent un matériau exceptionnel pour l'histoire de l'islam en général et de l'art islamique en particulier, non seulement en péninsule malaise mais aussi dans toute l'aire de distribution de cette famille de stèles. Les données épigraphiques à caractère historique encore lisibles indiquent, quant à elles, que ces monuments étaient réservés aux sultans, aux grands personnages musulmans des sultanats ainsi qu'à leurs proches (4). C'est ici sur le plan de l'histoire politique des sultanats que les batu Aceh peuvent se révéler des sources de première importance.

Des descriptions précises et systématiques réalisées au cours de ces récentes prospections en péninsule malaise permettent de proposer aujourd'hui une nouvelle classification qui fait l'objet du présent article.

\section{Les étapes de la recherche sur les batu Aceh en Malaisie}

L'évolution de la recherche sur les batu Aceh de Malaisie peut être divisée en trois périodes. Les premières investigations se déroulent durant la

3. Il s'agit du passage relatant l'envoi par le sultan d'Aceh, Iskandar Thani, de stèles pour des tombes situées à Pahang (Nuruddin al-Raniri, Bustan al-Salatin, Bab. II. Fasal 13. Siti Hawa Haji Salleh (éd.), Kuala Lumpur, Dewan Bahasa dan Pustaka, 1992, pp. 40-42).

4. D'une manière générale, les sources locales anciennes renseignent peu sur les pratiques funéraires. Il faut ainsi se tourner vers une source chinoise de la première moitié du XVI siècle pour apprendre que les habitants de Melaka, riches ou pauvres, brûlent leurs cadavres, avec pour seule différence que les cadavres des riches sont placés préalablement dans un cercueil avec du camphre (W.P. Groeneveldt, Notes on the Malay Archipelago and Malacea Compiled from Chinese Sources, Verhandelingen van de Bataviaasch Genootschap 39, 1880, p. 128). Cette observation est confirmée à Johor par Chang Hsich qui, dans son T'ung Hsi Yang $K^{\prime}$ (ao (1573-1619), note que tous les cadavres sont brûlés (Han Wai Toon, «A Study on Johore Lama». Joumal of the South Seas Society, 5(2). 1948, p. 31). Il semble qu'il faille attendre le XVI ${ }^{\mathrm{e}}$ siècle, voire le début du XVIII ${ }^{\mathrm{e}}$ siècle, pour que l'usage de la tombe marquée par des stèles en pierres brutes ou en bois se répande véritablement. En effet, Le Hai-lı, une source chinoise de la fin du XVIJC siècle, ne mentionne plus de crémations mais la présence de tombes chez les Malais de la côte orientale de la péninsule Malaise, y compris à 
période coloniale, principalement durant les décennies 1920 et 1930. Trois chercheurs sont particulièrement actifs dans le domaine : Richard Olaf Winstedt à Johor, William Linehan à Pahang et Engku 'Abdu'l-Hamid bin 'Abdu'l-Majid pour l'épigraphie. Leurs travaux paraissent dans le Journal of the Straits Branch of the Royal Asiatic Society (JSBRAS) qui devient en 1923 le Journal of the Malayan Branch of the Royal Asiatic Society (JMBRAS)(5). Intéressés surtout par l'histoire politique, ils privilégient les données épigraphiques à caractère historique et s'en tiennent à des considérations morphologiques générales pour la description des stèles (6). Leurs contributions sont toutefois essentielles, aussi bien par des détails qui permettent de retrouver aujourd'hui certains monuments et par la qualité des études épigraphiques, que par les documents photographiques relativement nombreux et de bonne qualité qu'ils ont publiés.

Le second moment se situe durant les décennies 1970 et 1980 avec les travaux de Abdul Halim Nasir et Othman bin Mohd. Yatim, à l'époque employés au service des Antiquités du Muzium Negara à Kuala Lumpur. De par leur fonction, ils sillonnent alors la péninsule, retrouvent de nombreux sites oubliés depuis la période coloniale et repèrent des tombes inédites. Les données de ces prospections serviront de matériau à la première thèse de doctorat sur les batu Aceh de Malaisie, thèse soutenue par Othman bin Mohd. Yatim à l'université de Durham en 1985 (7). C'est dans cette thèse que l'auteur suggère la première typologie des batu Aceh de péninsule malaise (8), formulant également des hypothèses sur l'évolution chronologique de chacun des types ainsi que sur le symbolisme des formes et des décors.

Pattani (J.W. Cushman \& A.C. Milner, «Eighteenth and nineteenth-century Chinese accounts of the Malay Peninsula", Journal of the Malaysian Branch of the Royal Asiatic Society, 52(1), 1979, pp. 13, 15).

5. R.O. Winstedt, "The Tomb of Mansur Shah, Sultan of Malacca, 1459-? 1475 A.D.", JSBRAS, 78, 1918, pp. 46-48, I pl.; "Muslim Tombstones in Raffles Museum », JMBRAS, X(1), 1932, pp. 6-8, 4 pl.; «A History of Johore (1365-1895 A.D.)», JMBRAS, X(3), 1932, pp. 1-158. W. Linehan, "Tomb-Stones of Muhammad Shah I of Pahang », JMBRAS, IV(2), 1926, pp. 188-192, 2 pl.; «Marhum Muda Pahang», JMBRAS, XII(2), 1934, pp. 171-173, 2 pl.; «A History of Pahang", JMBRAS, XIV(2), 1936, pp. 1-257, pl. VII-XIV. Engku 'Abdu'l-Hamid bin Engku 'Abdu'I-Majid, "Inscriptions on ancient Johor grave-stones », in R.O. Winstedt, «A History of Johore (1365-1895 A.D.)», JMBRAS, X(3), 1932, pp. 159-167, pl. II-XXXIV.

6. Ils emploient des expressions telles que «Malayo-Achinese type», «inferior specimens of the elaborately foliate type», «European-lantern (or "pine-apple') type», «usual XVIIth century type» ou encore «Chinese-lantern type».

7. Sous le titre Batu Aceh : a study of 15th-19th century Islamic gravestones in Peninsular Malaysia. Cette thèse est publiće trois ans plus tard par le Muzium Negara à Kuala Lumpur sous le titre Batu Aceh : Early Islamic Gravestones in Peninsular Malaysia.

8. Il distingue quatorze types d'après la forme générale (Othman bin Mohd. Yatim. op. cit.. 1988. pp. 52-58). Il faut noter qu'un an auparavant, également dans une thèse de doctorat, Hasan Muarif Ambary propose une typologie. il est vrai plus sommaire, à partir d'un corpus de batu Aceh d'Indonésie et de Malaisie (L'art funéraire musulman en Indonésie des origines au XIX" siècle. Étude épigraphique et typologique, Paris, EHESS). 
La troisième étape débute en 1996 à l'initiative des auteurs du présent article. L'idée de base est de réaliser un inventaire aussi complet que possible des batu Aceh de péninsule malaise (9) comprenant une localisation précise des tombes $(10)$ ainsi qu'une description systématique et détaillée de la stèle dans le meilleur état pour chaque monument(11). Ces descriptions débouchent sur l'établissement d'une classification basée sur la forme générale des stèles et sur la production d'un corpus des éléments décoratifs. Ces deux outils peuvent à terme être utilisés pour de multiples travaux comparatifs visant au repérage de la distribution d'une forme ou d'un élément décoratif sur toute l'aire de diffusion de ces stèles afin de mettre en lumière par exemple l'existence d'ateliers distincts. Au volet proprement descriptif du programme s'ajoute un volet épigraphique comportant non seulement la lecture des inscriptions nouvelles mais également la relecture des inscriptions déjà publiées(12). À son tour, le croisement des données purement descriptives et des données épigraphiques doit permettre la formulation d'hypothèses sur l'évolution chronologique des formes, sur leur rapport éventuel avec la position sociale des défunts, voire, dans le meilleur des cas, sur l'identification de certaines tombes.

Le bilan quantitatif de cette dernière étape montre une très nette augmentation du nombre de monuments par rapport aux deux étapes précédentes (cf. tableau ci-dessous). Si la majorité des monuments connus auparavant ont pu être retrouvés, les prospections les plus récentes ont révélé un grand nombre de tombes inédites. Les 450 tombes recensées sont réparties sur 123 sites. Presque les deux tiers de ces sites ne comportent qu'un ou deux monuments, alors que le cimetière le plus riche en batu Aceh est celui de Ulu Sungai Che Omar à Johor (24 tombes) (13). Cinq États abritent $95 \%$ des monuments : Johor, Kedah, Pahang, Perak et Melaka. À lui seul, l'État de Johor en totalise

9. À notre connaissance, aucune batu Aceh n'a pour l'instant été repérée à Sarawak et à Sabah.

10. Coordonnées topographiques obtenues par GPS.

11. Dans les rares cas où une tombe présente aujourd'hui deux stèles différentes, chaque stèle fait l'objet d'une description séparée. D'après Winstedt, il pourrait s'agir de deux monuments distincts (Winstedt, op. cit., 1932, p. 215). Il est clair par ailleurs que de nombreuses stèles ont été déplacées, notamment pour être installées dans des bâtiments commémoratifs et que d'autres ont été arrachées. Ainsi, la position actuelle des stèles peut ne pas refléter l'agencement originel.

12. Ce bilan, qui concerne 56 stèles, dont 18 comportent des inscriptions à caractère historique, est effectué par Ludvik Kalus, professeur au Département d'Études Arabes et Orientales, Université Paris IV-Sorbonne.

13. Mentionné par Winstedt en 1932 puis dans une publication locale en 1950, ce site n'a été décrit pour la première fois qu'en 1998 (Cf. Daniel Perret et Kamarudin Ab. Razak, Kompleks Makam Ulu Sungai Che Omar dan Kompleks Makam Bukit Telepok, Johor Bahru, École française d'Extrême-Orient, Yayasan Warisan Johor). 
près de la moitié, alors que la côte orientale de la péninsule, y compris à Johor mais à l'exception de Pahang, est très pauvre en batu Aceh. Il va de soi que ce nouvel inventaire n'est qu'un état de la question et ne saurait prétendre à l'exhaustivité(14). Fréquemment des tombes sont en effet découvertes par hasard, en particulier à l'occasion des multiples et extensifs aménagements du paysage qui transforment aujourd'hui la péninsule, qu'il s'agisse de plantations, de lotissements ou encore de zones industrielles.

Sur le plan descriptif, le doublement du corpus amène à repenser la classification proposée par Othman bin Mohd. Yatim il y a près de vingt ans. C'est ce que nous allons exposer dans la suite. Par ailleurs, l'enregistrement minutieux des éléments décoratifs a permis la constitution d'un corpus de plus de 400 traits, reflétant à la fois la richesse et la variété des décors des batu Aceh. Pour preuve, au stade actuel de l'enquête, il n'existe pas deux monuments identiques en péninsule malaise.

\section{Évolution du nombre de monuments recensés}

\begin{tabular}{|c|c|c|c|}
\hline État & EFEO/YWJ & OTHMAN & Pér. Col. \\
\hline Johor & 215 & 102 & 137 \\
\hline Kedah & 79 & 30 & 4 \\
\hline Pahang & 49 & 39 & 15 \\
\hline Perak & 44 & 24 & 8 \\
\hline Melaka & 39 & 2 & 2 \\
\hline Perlis & 6 & 8 & 0 \\
\hline Negeri Sembilan & 3 & 0 & 0 \\
\hline Pulau Pinang & 2 & 0 & 0 \\
\hline Terengganu & 2 & 3 & 0 \\
\hline Kelantan & 1 & 0 & 1 \\
\hline Heritage Cons. Cent. Singapour & 3 & 3 & 3 \\
\hline Musée Nat. Kuala Lumpur & 7 & 0 & \\
\hline Total & 450 & 211 & 170 \\
\hline
\end{tabular}

\section{Essai de classification}

Le principe qui régit notre description des batu Aceh est leur division en six parties maximum : base, partie inférieure du corps, partie supérieure du

14. Une monographie consacrée aux batu Aceh de l'État de Johor est parue en 1999 (Cf. Daniel Perret, Kamarudin Ab. Razak \& Ludvik Kalus, Batu Aceh: Warisan Sejarah Johor, Johor Bahru, École française d'Extrême-Orient, Yayasan Warisan Johor). Celle consacrée aux batu Aceh des autres États de la péninsule malaise paraîtra fin 2003 (Cf. Daniel Perret, Kamarudin Ab. Razak \& Ludvik Kalus, Batı Aceh dari Johor dalam Perbandingan, Johor Bahru, École française d'Extrême-Orient, Yayasan Warisan Johor). 
corps, épaules, tête et sommet(15). La partie supérieure du corps étant la seule qui se retrouve immanquablement sur toutes les stèles(16), c'est logiquement celle-ci que nous retenons pour établir la classification. La forme de la section de cette partie permet de distinguer cinq groupes de batu Aceh en péninsule malaise : le groupe «dalle», le groupe «carré», le groupe «tronconique inversé à huit faces », le groupe «octogonal à quatre épaules», le groupe «tronconique inversé simple». Dans chaque groupe, nous avons identifié un certain nombre de formes dont la distinction repose sur l'absence ou la forme spécifique de telle ou telle partie ou encore sur la présence de tel élément décoratif. Le groupe «dalle» comprend onze formes : A1, A2, B, C, D, $\mathrm{E} 1, \mathrm{E} 2, \mathrm{~F}, \mathrm{G}, \mathrm{H} 1, \mathrm{H} 2$. Le groupe «carré» comprend trois formes : I, J, K. Le groupe «tronconique inversé à huit faces» comprend quatre formes : L, M, $\mathrm{N}$, O. Les groupes «octogonal à quatre épaules» et «tronconique inversé simple » comprennent une forme, respectivement $\mathrm{P}$ et $\mathrm{Q}$. Les caractéristiques principales de chaque forme sont les suivantes :

\section{Forme A}

Le critère principal d'identification de cette forme est une partie supérieure du corps rectiligne d'une largeur constante, une tête de forme bilobée à pointe tronquée et l'absence de sommet. La base est rectangulaire, ainsi que la partie inférieure du corps, elle-même ornée de motifs polylobés ou d'entrelacs. La partie supérieure du corps est souvent décorée d'un panneau, dont seul le cadre est marqué, avec un motif polylobé en relief à la base. Cette partie présente aussi parfois plusieurs registres à inscriptions superposés. La tête peut être décorée d'un registre inscrit ou encore de motifs divers. A2 se distingue de A1 par le fait que la partie supérieure du corps et la tête du premier ne sont jamais décorées.

\section{Forme $B$}

Le critère principal d'identification de cette forme est une partie supérieure du corps rectiligne s'élargissant progressivement vers le haut, associée à une tête de forme bilobée à deux niveaux. La base est rectangulaire sans décor. La partie supérieure du corps est ornée d'un panneau sculpté en creux. Le sommet est trapézoïdal.

15. Cette partition est un peu différente de celle proposée par Othman bin Mohd. Yatim qui ne distingue pas deux parties dans le corps (Cf. Othman bin Mohd. Yatim, op. cit., 1988, pp. 26-27).

16. Sauf bien évidemment sur des stèles cassées à ce niveau. 


\section{Forme C}

Le critère principal d'identification de cette forme est une partie supérieure du corps au profil curviligne, s'élargissant vers le haut, associée à une tête de forme bilobée. La base est absente ou rectangulaire sans décor. Le sommet est trapézoïdal.

\section{Forme D}

Le critère principal d'identification de cette forme est l'épaule courte recourbée vers le haut qui prolonge perpendiculairement le profil en haut d'un corps surmonté d'une tête à un niveau. Cette épaule est généralement ornée, à la base, par une excroissance bilobée recourbée vers le bas. La base, de forme rectangulaire, est souvent décorée de motifs polylobés inversés ou de panneaux rectangulaires en creux. La partie supérieure du corps est décorée d'un panneau orné soit d'un motif polylobé en relief à la base, soit de registres inscrits superposés. La tête, de forme bilobée, est fréquemment décorée d'un panneau avec un motif polylobé en relief. Le sommet est trapézoïdal ou en pyramide tronquée.

\section{Forme $E$}

Le critère principal d'identification de cette forme est la tête bilobée qui s'évase vers le haut sur un corps qui s'élargit du bas vers le haut. La base, de forme rectangulaire, compte un ou plusieurs panneaux rectangulaires en creux. La partie inférieure du corps, de forme trapézoïdale, est ornée de motifs polylobés en relief. Le décor le plus fréquent de la partie supérieure du corps consiste en trois panneaux à inscriptions «schématisées» formant une échelle. La tête est ornée d'un panneau central en creux généralement aussi à décor d'inscription «schématisée ». E2 se distingue de E1 par la présence d'un sommet à un niveau sur le premier.

\section{Forme $F$}

Le critère principal d'identification de cette forme est une partie supérieure du corps rectiligne d'une largeur constante, sans excroissances, surmontée d'une tête à deux niveaux : un niveau inférieur de forme bilobée et un niveau supérieur de forme triangulaire. La base, de forme rectangulaire, compte un ou plusieurs panneaux rectangulaires en creux. La partie inférieure du corps est ornée le plus souvent de motifs polylobés en relief alors que sa partie supérieure comporte généralement des panneaux en creux à inscriptions «schématisées » formant une échelle. Le sommet comporte un seul niveau.

\section{Forme $G$}

Le critère principal d'identification de cette forme est l'excroissance qui prolonge verticalement la partie supérieure du corps sur les petits côtés. La 
base, de forme rectangulaire, compte un ou plusieurs panneaux rectangulaires en creux. Le corps s'élargit légèrement du bas vers le haut. Sa partie inférieure est ornée le plus souvent de motifs polylobés en relief. Sa partie supérieure comporte généralement des panneaux en creux à inscriptions «schématisées » formant une échelle, ainsi qu'un panneau en creux à inscriptions «schématisées» de part et d'autre de cette échelle. La tête présente deux niveaux plus ou moins ornés : un niveau inférieur de forme bilobée et un niveau supérieur de forme triangulaire. Le sommet comporte un seul niveau fréquemment orné d'une inscription «schématisée».

\section{Forme $H$}

Le critère principal d'identification de cette forme est l'épaule recourbée vers le haut, le plus souvent polylobée, qui prolonge perpendiculairement le profil en haut du corps surmonté d'une tête à deux niveaux. La base, de forme rectangulaire, compte un ou plusieurs panneaux rectangulaires en creux. La partie inférieure du corps est ornée le plus souvent de motifs polylobés en relief. Sa partie supérieure comporte généralement une échelle dont le contenu varie : panneaux en creux, niveaux d'inscriptions «schématisées», motifs floraux, entrelacs, etc. La tête présente deux niveaux plus ou moins ornés : un niveau inférieur de forme bilobée et un niveau supérieur de forme triangulaire. Le sommet comporte un seul niveau. Ce qui distingue $\mathrm{H} 2 \mathrm{de} \mathrm{H} 1$ est la présence, sur $\mathrm{H} 2$, de rangées d'excroissances bilobées ou triangulaires sur les profils des épaules, de la tête et du sommet. Par ailleurs, la plupart des stèles $\mathrm{H} 2$ présentent une ligne verticale en relief au centre de chaque grande face, de la base du corps au sommet.

\section{Forme I}

Le critère principal d'identification de cette forme est l'excroissance bilobée qui prolonge verticalement chaque angle de la partie supérieure du corps surmonté d'un sommet. La base carrée compte plusieurs panneaux rectangulaires en creux. La partie inférieure du corps est ornée le plus souvent de motifs polylobés en relief, alors que sa partie supérieure comprend généralement trois panneaux en creux à inscriptions «schématisées» formant une échelle. Le sommet comporte quatre niveaux.

\section{Forme $J$}

Le critère principal d'identification de cette forme est l'épaule courte, à décors d'entrelacs, pointée vers le haut à chaque angle, entre la partie supérieure du corps et le sommet. La base carrée compte plusieurs panneaux rectangulaires en creux. La partie inférieure du corps est ornée le plus souvent de motifs polylobés en relief. Sa partie supérieure comprend plusieurs panneaux rectangulaires en creux formant une échelle. Elle reçoit également les 
supports des épaules à chaque angle. Le sommet comporte trois ou quatre niveaux.

\section{Forme $K$}

Le critère principal d'identification de cette forme est l'épaule perpendiculaire à l'axe vertical de la stèle, polylobée et recourbée vers le haut à chaque angle, entre la partie supérieure du corps et le sommet. La base carrée compte plusieurs panneaux rectangulaires en creux. La partie inférieure du corps est ornée le plus souvent de motifs polylobés en relief. Sa partie supérieure comporte plusieurs panneaux rectangulaires en creux formant une échelle, qui contiennent généralement des inscriptions «schématisées", mais parfois de véritables inscriptions. Elle reçoit également les supports des épaules à chaque angle. Le sommet comporte de quatre à six niveaux.

\section{Forme $L$}

Le critère principal d'identification de cette forme est l'absence de décor sur la partie supérieure du corps, où chacune des huit faces comporte deux panneaux plats inclinés vers l'intérieur. La base carrée compte un ou plusieurs panneaux en creux. La partie inférieure du corps, de forme octogonale, est souvent ornée d'un motif polylobé en relief à l'extrémité de chacune des faces. La tête est constituée d'un ou deux disques superposés à décor de pétales de lotus. Le sommet, à un seul niveau, est un volume bulbeux octogonal allongé dans sa partie supérieure.

\section{Forme M}

Le critère principal d'identification de cette forme est la présence, sur l'arête intérieure de chacune des huit faces de la partie supérieure du corps, d'une excroissance sans décor. La base carrée compte un ou plusieurs panneaux en creux. La partie inférieure du corps, de forme octogonale, est ornée d'un motif polylobé en relief à l'extrémité de chacune des faces. La tête est constituée d'un ou deux disques superposés à décor de pétales de lotus. Le sommet, à un seul niveau, est un volume bulbeux octogonal très allongé dans sa partie supérieure.

\section{Forme N}

Le critère principal d'identification de cette forme est la présence, au centre de chacune des huit faces de la partie supérieure du corps, d'une excroissance verticale décorée. Si la majorité des stèles de ce type comportent des faces à deux panneaux plats inclinés vers l'intérieur, on observe également des faces à deux panneaux arrondis ainsi que des faces plates. La base carrée compte un ou plusieurs panneaux en creux. La partie inférieure du corps, de forme octogonale, est ornée d'un motif polylobé en relief à 
l'extrémité de chacune des faces. Dans le haut du corps, l'extrémité de chaque face est parfois prolongée par une excroissance verticale bilobée. La tête est constituée d'un ou deux disques superposés à décor de pétales de lotus. Le sommet présente de un à quatre niveaux.

\section{Forme $O$}

Le critère principal d'identification de cette forme est l'absence de l'excroissance verticale centrale sur chacune des huit faces plates de la partie supérieure du corps. Dans la plupart des cas, chaque face est ornée d'un panneau sculpté en creux. Celui-ci porte généralement une inscription ou un décor d'inscriptions «schématisées». La base carrée compte un ou plusieurs panneaux en creux. La partie inférieure du corps, de forme octogonale, est ornée d'un motif polylobé en relief à l'extrémité de chacune des faces. Dans le haut du corps, l'extrémité de chaque face est très souvent prolongée par une excroissance verticale bilobée. La tête est constituée d'un ou deux disques superposés à décor de pétales de lotus. Le sommet présente de un à trois niveaux.

\section{Forme $P$}

$P$ étant la seule forme pour le groupe considéré, son critère principal d'identification se confond avec celui du groupe, à savoir une partie supérieure du corps octogonale surmontée de quatre épaules perpendiculaires à l'axe vertical de la stèle. La base carrée compte un ou plusieurs panneaux en creux. La partie inférieure du corps, de forme octogonale, est ornée d'un motif polylobé en relief à l'extrémité de chacune des faces. Sa partie supérieure reçoit les supports des épaules polylobées à décors d'entrelacs et recourbées vers le haut. Le sommet, qui s'appuie directement sur les épaules, comporte cinq niveaux.

\section{Forme $Q$}

$\mathrm{Q}$ étant la seule forme pour le groupe considéré, son critère principal d'identification se confond avec celui du groupe, à savoir une partie supérieure du corps tronconique inversée simple, c'est-à-dire sans faces. La base carrée compte un ou plusieurs panneaux en creux. La partie inférieure du corps, de forme octogonale, est ornée d'un motif polylobé en relief à l'extrémité de chacune des faces. Le sommet a la forme d'un entonnoir renversé.

\section{Les apports de la classification}

Les données collectées à l'occasion des plus récentes prospections sont en cours d'exploitation. On peut néanmoins tirer les premiers enseignements généraux des résultats et fournir un exemple de l'aide apportée par la classification des batu Aceh à l'interprétation historique.

En péninsule malaise, c'est le groupe en forme de dalle qui prédomine. 


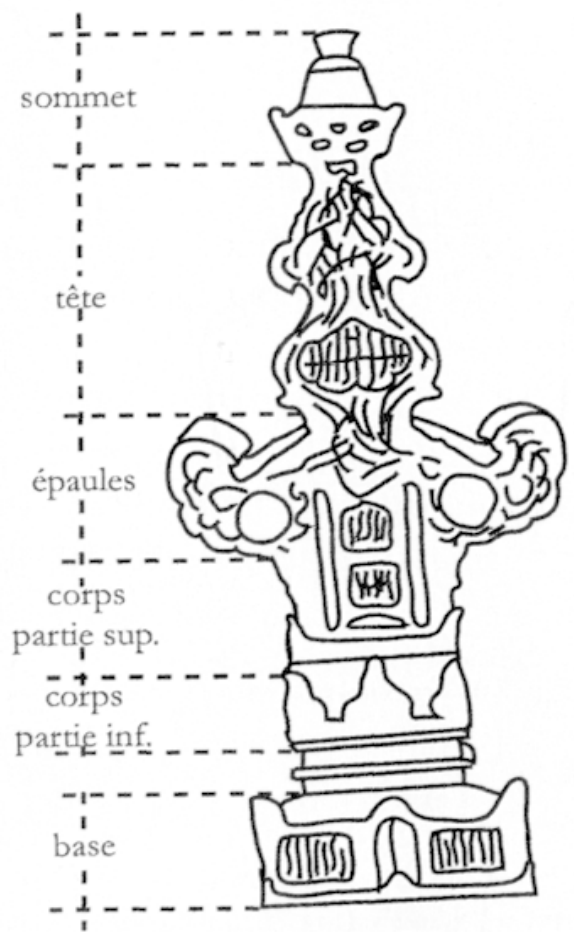

Forme $\mathbf{H}$

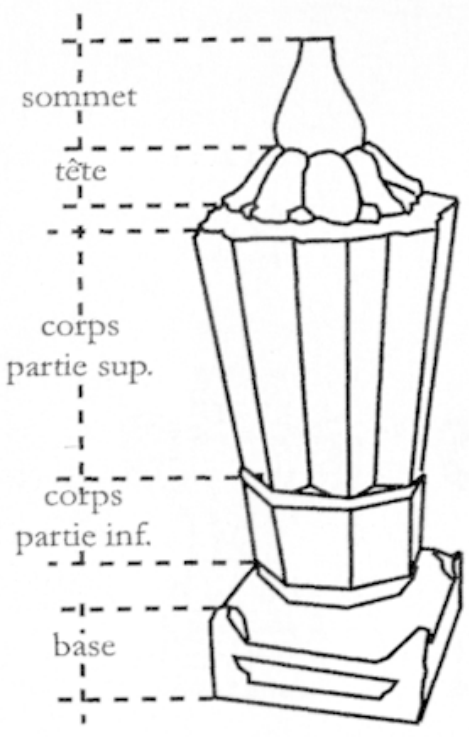

Forme L

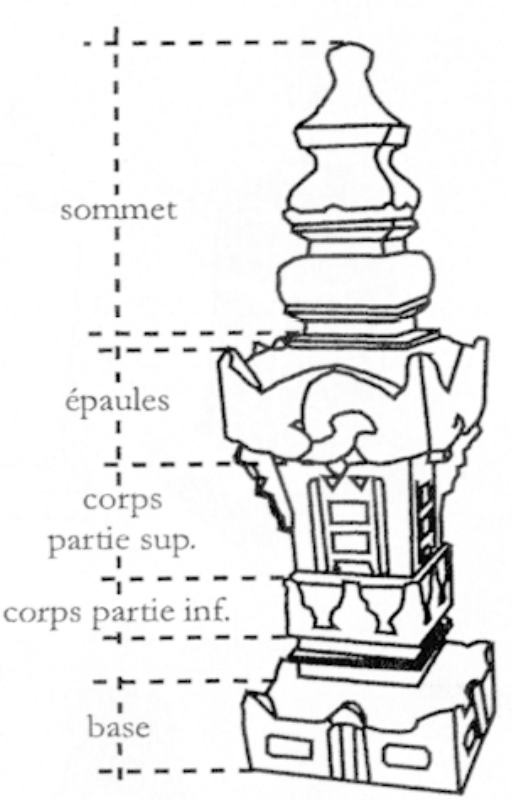

Forme J

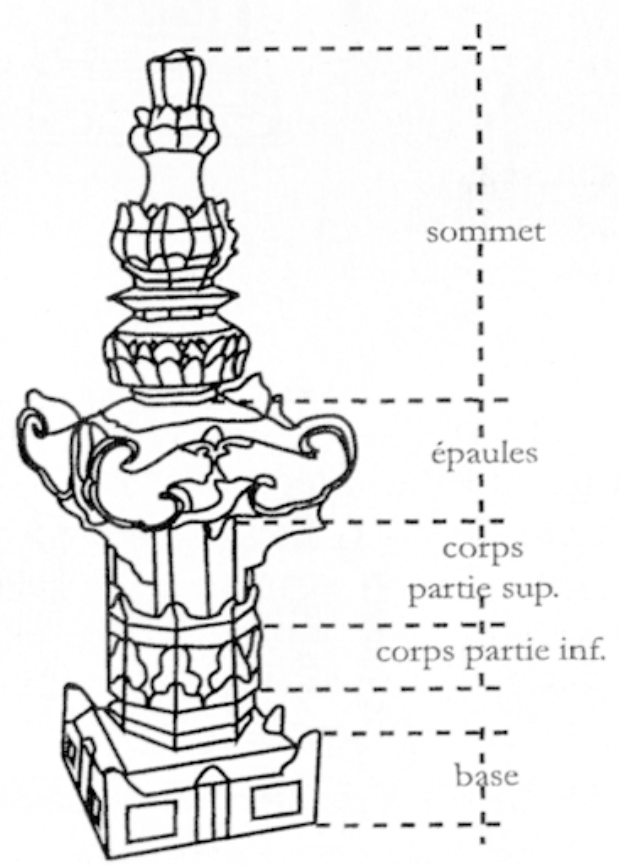

Forme P

Exemples de partition de stèles 

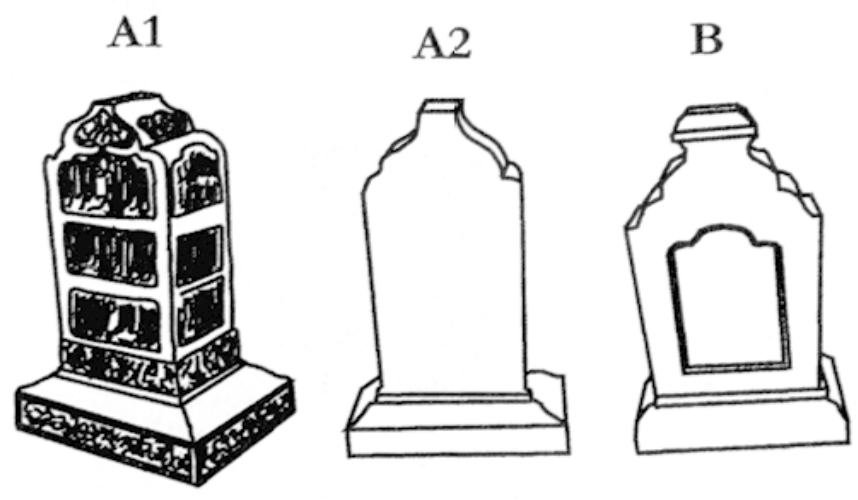

C
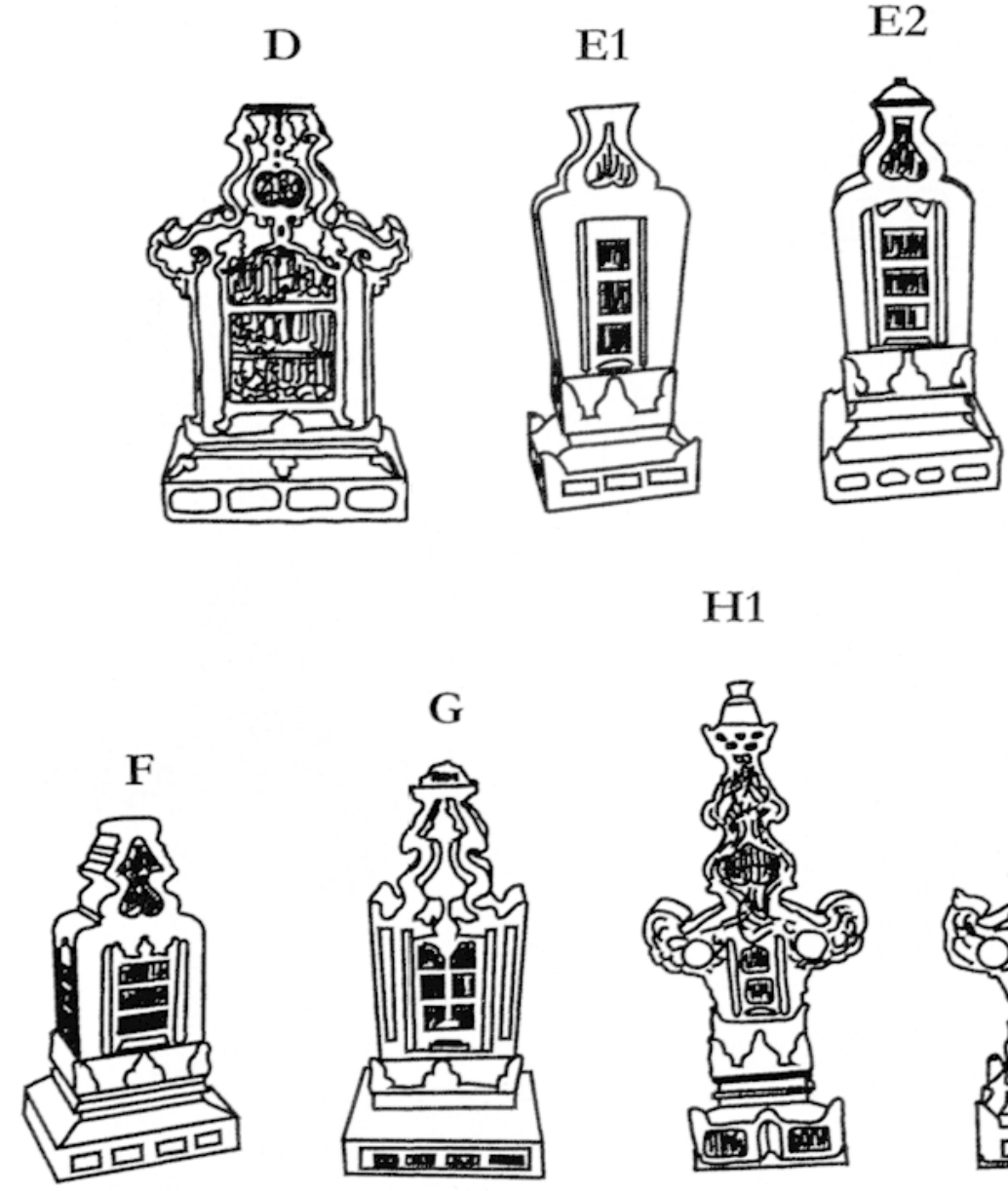

H1

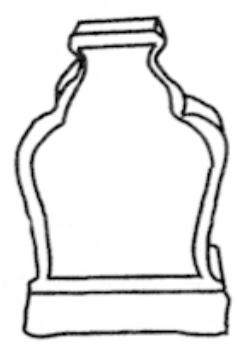

2 

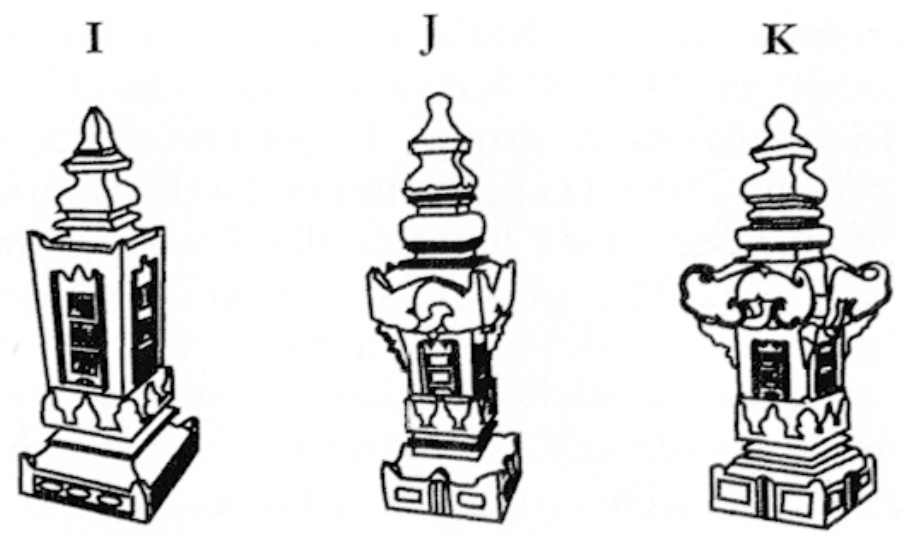

Stèles de section carrée

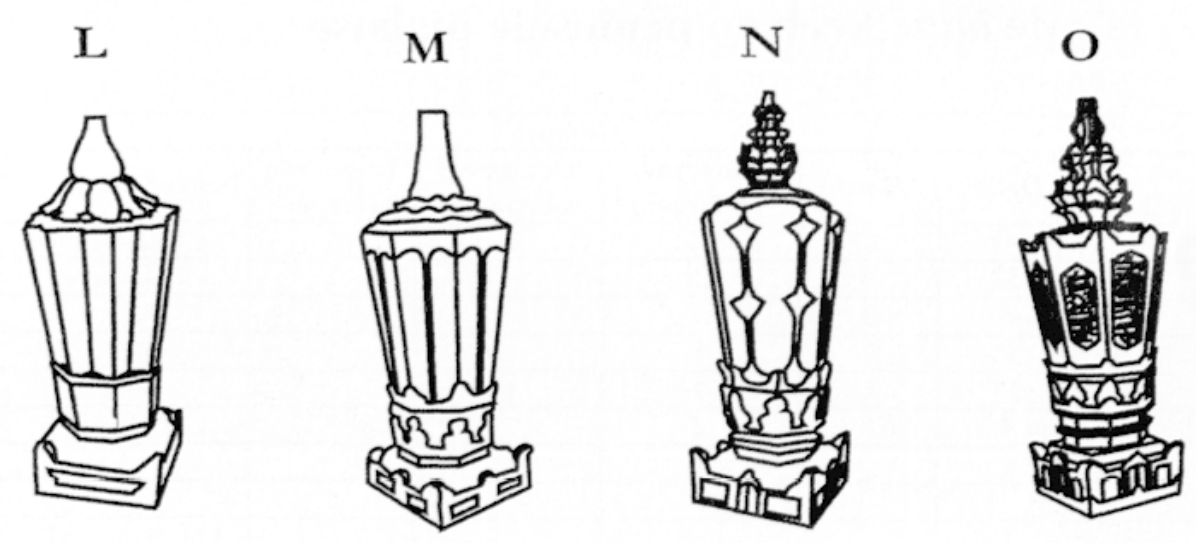

Stèles de forme tronconique inversée à huit faces

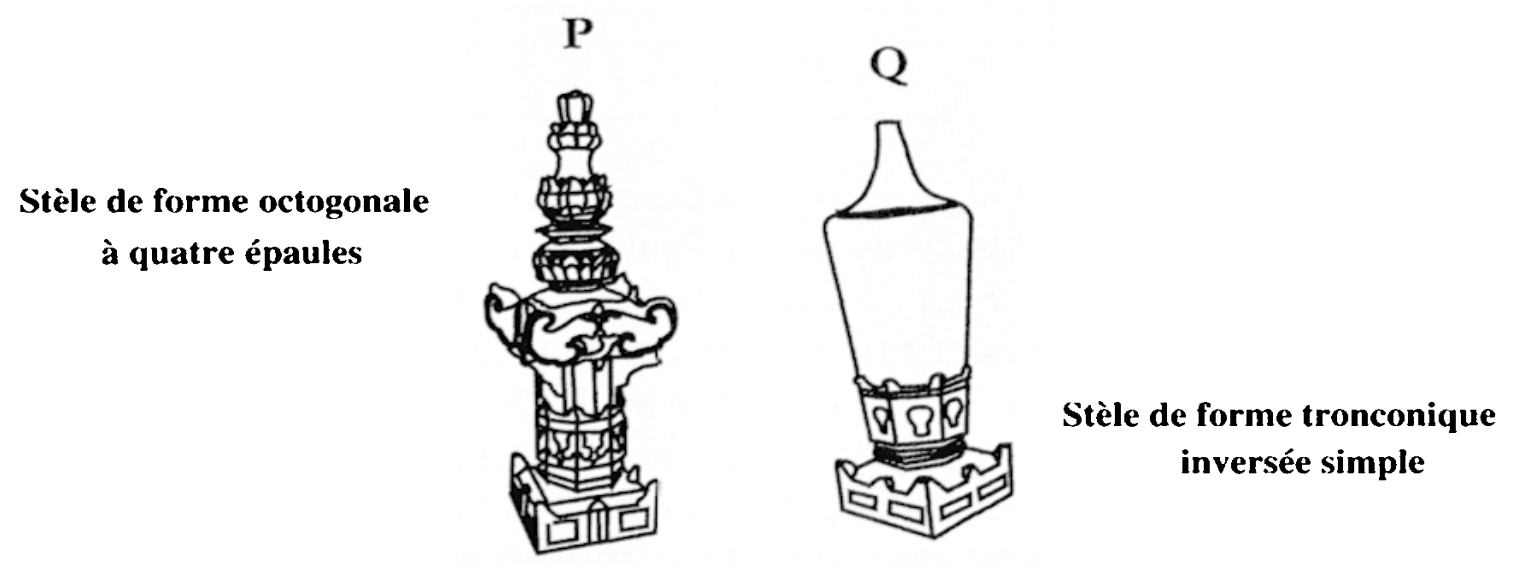


On l'observe sur près de deux tiers des monuments recensés et il est présent dans neuf des dix États où des batu Aceh ont été repérées. Dans les cinq États les plus riches en batu Aceh de Malaisie, le poids relatif de ce groupe oscille entre $47 \%$ (Kedah) et $84 \%$ (Pahang). Par ailleurs, 43 des 56 stèles inscrites de la région font partie de ce groupe. Le groupe tronconique inversé à huit faces suit avec près de $20 \%$ des monuments. Sa distribution est également large, puisqu'il est présent dans huit des dix États à batu Aceh. Son poids relatif dans les cinq États les plus riches en batu Aceh est beaucoup plus irrégulier que le groupe précédent : de moins de $1 \%$ à Perak à $42 \%$ à Kedah. Il totalise par ailleurs six stèles inscrites. Le groupe carré ne représente que $6 \%$ des monuments recensés et s'observe dans quatre États seulement. Il compte toutefois sept stèles inscrites. Johor est le seul État où tous les groupes classificatoires sont représentés alors qu'un ou deux groupes manquent dans les quatre autres États les plus fournis.

\section{Distribution géographique des groupes classificatoires de batu Aceh en péninsule malaise}

\begin{tabular}{|c|c|c|c|c|c|c|c|}
\hline \multirow[b]{2}{*}{ Lieu } & \multicolumn{6}{|c|}{ Groupes } & \multirow[b]{2}{*}{ Total } \\
\hline & Dalle & Carré & $\begin{array}{l}\text { Tronc. inv. } \\
8 \text { faces }\end{array}$ & $\begin{array}{l}\text { Octogonal } \\
\text { à } 4 \text { épaules }\end{array}$ & $\begin{array}{l}\text { Tronc. inv. } \\
\text { simple }\end{array}$ & Non identifié & \\
\hline Johor & 141 & 13 & 36 & 3 & 3 & 23 & 219 \\
\hline Kedah & 37 & 4 & 33 & & 1 & 4 & 79 \\
\hline Pahang & 41 & 3 & 3 & & & 2 & 49 \\
\hline Perak & 32 & 7 & 1 & & 1 & 3 & 44 \\
\hline Melaka & 28 & & 9 & 1 & & 2 & 40 \\
\hline Perlis & 4 & & 2 & & & & 6 \\
\hline Negeri Sembilan & 1 & & 2 & & & & 3 \\
\hline Pulau Pinang & & & 1 & & 1 & & 2 \\
\hline Terengganu & 1 & & & & & 1 & 2 \\
\hline Kelantan & 1 & & & & & & 1 \\
\hline Heritage Cons. Cent. & 3 & & & & & & 3 \\
\hline Musée Nat. Kuala Lumpur & 6 & & 1 & & & & 7 \\
\hline & & & & & & & \\
\hline Total & 295 & 27 & 88 & 4 & 6 & 35 & 455 \\
\hline Pourcentage & 65 & 6 & 19 & 1 & 1 & 8 & 100 \\
\hline
\end{tabular}

C'est de loin $\mathrm{H} l$ qui est le mieux représenté en péninsule malaise avec $35 \%$ des monuments. Sa distribution est également la plus large avec une présence dans sept des dix États à batu Aceh. Toutefois, son poids relatif varie beaucoup parmi les cinq États les plus riches en batu Aceh : de $1 \%$ à Pahang à $51 \%$ à Melaka. $M$ vient ensuite avec $10 \%$ des monuments et une présence dans six États. Son poids relatif ne dépasse pas $15 \%$ (Melaka). Derrière $\mathrm{H} 1$ et $M$, cinq formes sont présentes dans cinq États (A1, D, G, H2, N). On remarquera également que plus de la moitié des formes sont représentées dans quatre États avec la plus grande variété pour Johor, où l'on peut observer 18 des 20 formes. Enfin, cinq formes sont ornées d'inscriptions : A 1 (20), D (17), K (7), H1 (6), O (6). 


\section{Distribution géographique des formes de batu Aceh en péninsule malaise}

\begin{tabular}{|c|c|c|c|c|c|c|c|c|c|c|c|c|c|c|c|c|c|c|c|c|c|c|}
\hline & \multicolumn{21}{|c|}{ Formes } & \multirow[b]{2}{*}{ Total } \\
\hline Lieu & A1 & $\mathrm{A} 2$ & $\mathrm{~B}$ & $\mathrm{C}$ & $\mathrm{D}$ & E1 & $\mathrm{E} 2$ & $\mathrm{~F}$ & G & H1 & $\mathrm{H} 2$ & I & $\mathrm{J}$ & $\mathrm{K}$ & $\mathrm{L}$ & M & $\mathrm{N}$ & $\mathrm{O}$ & $\mathrm{P}$ & Q & $?$ & \\
\hline Johor & 4 & 9 & & 1 & 19 & 8 & 2 & 4 & 6 & 84 & 4 & 1 & 6 & 6 & 4 & 25 & 7 & & 3 & 3 & 23 & 219 \\
\hline Kedah & 1 & & & & & 1 & 3 & & 1 & 24 & 7 & 1 & 2 & 1 & 2 & 8 & 20 & 3 & & 1 & 4 & 79 \\
\hline Pahang & 7 & 13 & 1 & 1 & 6 & 1 & & 5 & 2 & 4 & 1 & & & 3 & & 2 & & 1 & & & 2 & 49 \\
\hline Perak & & 1 & & & 1 & & & 3 & 5 & 21 & 1 & 1 & 5 & 1 & & 1 & & & & 1 & 3 & 44 \\
\hline Melaka & 1 & & & & 2 & & 1 & & & 20 & 4 & & & & & 6 & 3 & & 1 & & 2 & 40 \\
\hline Perlis & & & & & & & & & 2 & 2 & & & & & & 2 & & & & & & 6 \\
\hline Negeri Sembilan & & & & & & & & & & 1 & & & & & & & 2 & & & & & 3 \\
\hline Pulau Pinang & & & & & & & & & & & & & & & & & 1 & & & 1 & & 2 \\
\hline Terengganu & 1 & & & & & & & & & & & & & & & & & & & & 1 & 2 \\
\hline Kelantan & & & & & 1 & & & & & & & & & & & & & & & & & 1 \\
\hline Heritage Cons. Cent. Sing. & 1 & & & & 1 & & & & & 1 & & & & & & & & & & & & 3 \\
\hline Musée Nat. Kuala Lumpur & & & & & 1 & 1 & & 1 & 1 & 2 & & & & & & & 1 & & & & & 7 \\
\hline Total & 15 & 23 & 1 & 2 & 31 & 11 & 6 & 13 & 17 & 159 & 17 & 3 & 13 & 11 & 6 & 44 & 34 & 4 & 4 & 6 & 35 & 455 \\
\hline Pourcentage & 3 & 6 & & & 7 & 2 & 1 & 3 & 4 & 35 & 4 & 1 & 3 & 2 & 1 & 10 & 7 & 1 & 1 & 1 & 8 & 100 \\
\hline
\end{tabular}

Dans l'étude sur les batu Aceh de Johor, la classification nous a aidés à formuler de solides hypothèses d'identification pour plusieurs tombes. C'est ainsi le cas pour la tombe de Kota Tinggi codifiée BEN 13 dans notre inventaire. Ce monument fait partie d'un cimetière de 22 tombes à batu Aceh (dont aucune ne porte d'inscription) qui, selon les traditions locales, abriterait la famille du bendahara du sultanat de Johor, Sri Maharaja Tun Habab Abdul Majid. BEN 13 serait justement la tombe du bendahara. Les textes malais Tuhfat al-Nafis et Peringatan Sejarah Negeri Johor indiquent que ce personnage meurt le 7 Muharram 1109 H.(17) (26 juillet 1697). BEN 13 est une tombe de forme rare en péninsule malaise, $P$. En effet, seuls quatre monuments de forme $\mathrm{P}$ y sont pour l'instant recensés : trois à Johor et un à Melaka. Sans preuve directe permettant d'identifier avec certitude le défunt enterré en BEN 13 comme étant le bendahara Sri Maharaja Tun Habab Abdul Majid, il faut s'orienter vers la tombe qui présente un maximum de similitudes avec BEN 13, car elle recèle peut-être des informations éclairantes.

Parmi les trois autres monuments du même type, c'est PAN 5, une tombe du village de Panchor, en aval de Kota Tinggi sur le fleuve Johor, dont la description se rapproche le plus de BEN 13. Sans inscription, la tombe ellemême ne livre pas directement le nom du défunt. Son identité est même l'objet de controverses, puisque pas moins de trois candidats, deux sultans de Johor et un bendahara, sont avancés par diverses traditions orales. Sur la base de diverses sources, nous avons montré ailleurs que, très probablement,

17. Raja Ali Haji, Tuhfat al-Nafis-Sejarah Melayu-Islam. W. Matheson-Hooker (éd.), 1991, p. 139; E.U. Kratz (éd.), Peringatan Sejarah Negeri Johor. Eine Malaiische Quelle zu Geschichte Johors im 18. Jahrhundert, Wiesbaden, Otto Harrassowitz, 1973, p. 44. 
les deux sultans ne sont pas enterrés à Panchor(18). La tradition qui veut que PAN 5 soit la tombe d'un bendahara nous paraît par contre beaucoup plus solide. Une source locale, le Peringatan Sejarah Negeri Johor, mentionne en effet que le bendahara Tun Mas Anom meurt le 29 mars 1708, à l'époque où Panchor est la capitale du sultanat de Johor(19). Ce bendahara est un frère cadet de Sultan Abdul Jalil IV (20), par conséquent l'un des fils du bendahara Tun Habab Abdul Majid. Parti du seul rapprochement de forme, on retrouve ainsi trois éléments objectifs (lien de parenté étroit, identité de fonction et proximité des dates de décès) qui expliquent cette similitude et rendent ainsi très plausibles la tradition attachée à BEN 13 et l'une des traditions attachées à PAN 5. Du même coup, BEN 21, la troisième tombe de forme $\mathrm{P}$ de Johor, située à quelques mètres de BEN 13, abrite très probablement un proche du bendahara Tun Habab Abdul Majid qui aurait occupé des fonctions importantes dans le sultanat de Johor au tournant du XVIII ${ }^{\mathrm{e}}$ siècle.

Le lien est par contre beaucoup moins évident entre ces trois monuments et $\mathrm{BBRG}$ II/1, la quatrième tombe de forme $\mathrm{P}$ connue en péninsule malaise, située sur les hauteurs de Melaka, dans le village de Taman Kerjasama Bukit Beruang. Les deux batu Aceh de cette tombe sont sans conteste les plus sophistiquées de Malaisie. Selon le panneau explicatif disposé à l'entrée du cimetière, elle abriterait un certain Datuk Samsudin, le second Capitaine de la communauté malaise de Melaka à l'époque hollandaise. Celui-ci aurait hérité la fonction de son père, Datuk Arom, devenu le premier capitaine des Malais en récompense de l'aide apportée aux Hollandais et aux troupes de Johor pour chasser les Portugais de Melaka en 1641. Enterré à quelques dizaines de mètres de son fils, dans un cimetière appelé keramat Guba Dato Haron, son nom véritable serait Tai Seck Tiong. Sans inscriptions, les stèles ne peuvent confirmer ces explications, mais sachant que ces personnages occupaient généralement leur fonction jusqu'à leur mort, BBRG II/1 pourrait tout à fait être daté du tournant du XVIII ${ }^{\mathrm{e}}$ siècle comme BEN 13, PAN 5 et probablement BEN 21.

Si ces premières analyses comparatives basées sur la classification, bien que limitées à la péninsule malaise, apportent déjà des résultats intéressants, il est évident qu'une question comme la chronologie des formes ne pourra être éventuellement éclaircie qu'après avoir enrichi le corpus. Le passage du Bustan al-Salatin cité plus haut (cf. note 3) relatant l'envoi par le sultan d'Aceh de stèles pour des tombes de Pahang conduit en effet à penser qu'il

18. D. Perret, Kamarudin Ab. Razak, L. Kalus, op. cit., 1999, p. 184.

19. E.U. Kratz (éd.), op. cit., 1973, p. 46; L.Y. Andaya, The Kingdom of Johore (1641-1728), Kuala Lumpur, Oxford University Press. 1975, p. 211.

20. Andaya, op. cit., 1975, p. 201. 
n'y a pas toujours adéquation entre la date de décès du défunt et la date de mise en place des stèles visibles aujourd'hui. Plusieurs décennies ont pu s'écouler entre les deux événements. Ainsi, la datation certaine d'une forme ne peut reposer sur une seule tombe bien datée. De même les hypothèses sur les liens entre la fonction ou le statut social du défunt et telle forme de batu Aceh ne pourront qu'être renforcées ou au contraire invalidées en multipliant les comparaisons. C'est pourquoi le travail d'inventaire et d'étude épigraphique systématiques déjà effectué ne prendra en réalité toute sa valeur qu'après avoir procédé de même dans la province d'Aceh, là où se trouvent la majorité des monuments. 